Journal de la Société des américanistes

Journal de la Société

des américanistes

NIMUENDAJU Curt, Les Indiens Palikur et leurs voisins

Philippe Erikson

(2) OpenEdition

Journals

Édition électronique

URL : https://journals.openedition.org/jsa/12766

DOI : 10.4000/jsa. 12766

ISSN : 1957-7842

Éditeur

Société des américanistes

Édition imprimée

Date de publication : 5 décembre 2009

Pagination : 312-314

ISSN : 0037-9174

Référence électronique

Philippe Erikson, " nimuendasu Curt, Les Indiens Palikur et leurs voisins », Journal de la Société des

américanistes [En ligne], 95-2 | 2009, mis en ligne le 11 mars 2010, consulté le 04 septembre 2022.

URL : http://journals.openedition.org/jsa/12766 ; DOI : https://doi.org/10.4000/jsa.12766

Ce document a été généré automatiquement le 4 septembre 2022

Tous droits réservés 


\title{
NIMUENDAJU Curt, Les Indiens Palikur et leurs voisins
}

\author{
Philippe Erikson
}

\section{RÉFÉRENCE}

NIMUENDAJU Curt, Les Indiens Palikur et leurs voisins, présentation et notes de Pierre Grenand, traduit de l'allemand par Wolfgang Steiner et Joëlle Lecler, Éditions du Comité des travaux historiques et scientifiques/Presses universitaires d'Orléans, coll. « Encyclopédie palikur », Paris/Orléans, 2008, 183 p., bibl., nombr. ill. N\&B, carte

1 Originellement publié en 1926, cet ouvrage de Curt Unkel (alias Nimuendaju) inaugure le volet palikur de la superbe série que promet d'être la collection Encyclopédies des peuples de Guyane, dirigée par Françoise Grenand. Catalogue d'exposition virtuelle plutôt que véritable monographie, ce livre est abondamment illustré de 46 figures, pour l'essentiel des photos noir et blanc de l'auteur représentant les gens et les objets dont il est question dans le texte, auxquelles s'ajoutent quelques cartes et croquis.

2 En raison de la brièveté de son séjour sur le terrain (cinq mois au total) et parce que ses bailleurs de fond s'intéressaient avant tout à la collecte d'objets, Nimuendaju a construit son ouvrage comme une juxtaposition de fiches, avec quarante micro-chapitres consacrés aux Palikur (pp. 32-139), vingt-neuf aux Indiens de l'Uaçá (pp. 140-157) et une courte vignette sur les Brésiliens du rio Curipy (pp.158-161). L'ouvrage se termine (pp. 162-178) par une série de brefs lexiques palikur, galibi du rio Uaçá, auruá et maraón, ce dernier ne comprenant que deux entrées - «bonjour » et « je suis un vieil homme » qui fleurent bon l'ethnographie de sauvetage. Relevons toutefois que Nimuendaju semble avoir surtout, sur le terrain, utilisé la langue créole.

3 L'essentiel du texte est consacré à une présentation générale des Palikur, suivant un plan à tiroirs d'un parfait classicisme. On trouve d'abord une présentation générale des gens et du milieu (pp. 32-62: l'histoire, l'ethnonyme, la région, la démographie, les clans, l'hygiène, la division des tâches). Suit une partie plus spécifiquement consacrée à la 
culture matérielle (pp.63-99: armes, navigation, poteries, calebasses, vannerie, filage, instruments ménagers, instruments de musique, arts plastiques, costume, parures) et aux activités de subsistance (pp. 100-105: agriculture, pêche, chasse, animaux domestiques). Viennent ensuite des développements sur la vie familiale et sociale (pp.106-113: caractère et morale, statut de la femme, mariage, naissance, nom), avant d'aborder des considérations plus abstraites sur les croyances et la vie cérémonielle (pp.114-124: rituels de puberté, funérailles, religion, astronomie, chamanisme, fêtes), puis de conclure sur l'organisation politique, les relations extérieures et une rapide description de la langue (pp. 131-139). Les chapitres sont très courts, le plus long étant celui consacré à la description des fêtes de boisson (pp. 124-130).

La deuxième partie, consacrée au colluvies gentium (p.142) constitué par les Indiens de l'Uaça, reprend le même plan, dans une optique comparative souvent prétexte à un laconisme renforcé. Le titre de la rubrique 8, par exemple, « Répartition du travail », est presqu'aussi long que le texte qui la compose: «Autant que j'ai pu le voir, elle est identique à celle des Palikur ». L'entrée " hygiène » se réduit à : "Sans être malpropres, les Indiens de l'Uaça ne sont pourtant pas aussi propres que les Palikur. Il y a souvent des poux et on les mange»(p.146). Des instruments de musique du Uaça, on apprend seulement que : "Ce sont les mêmes que ceux des Palikur, les sifflets en terre et en bois en moins. Les tambours et les violons fabriqués par les paysans brésiliens, et même l'accordéon, prennent le dessus» (p.46). On est loin de la description détaillée de la toilette des Palikur, qui va jusqu'à évoquer le lien entre les odeurs et les soins donnés aux mourants (p.61); loin aussi des cinq pleines pages consacrées à l'organologie arawak dans la partie précédente (pp. 85-89)...

Sa relative concision stylistique, alliée à une précision extrême dans le choix des termes employés, confère à cet ouvrage un charme dépouillé. Ceux qui ne le savaient pas déjà apprendront en lisant ce livre que la partie de la kuyeraha qui se met en bouche se nomme le «cuilleron» (p.84); qu'une pagaie comprend trois parties, la poignée, le manche et la pale (p.69) ; qu'une pièce du costume féminin peut s'appeler le " gorgerin » (p. 93) ; que la soupe d'amidon de manioc se prépare à partir de l'« empois », tandis que la farine se fait à partir des tubercules "rouis ", etc. À cet égard, saluons au passage la rigueur et la qualité de la traduction, exemplaire à un détail près : l'emploi du terme " compas ", au lieu de "boussole ", pour rendre l'allemand kompass (p. 104). Soulignons également la grande qualité de l'appareil critique qui complète l'ouvrage, sous forme d'une présentation générale (pp.10-24), d'une bibliographie complémentaire (pp. 181-183) et surtout de quelque 182 notes de bas de page d'une précision admirable signées Pierre Grenand, dont le travail vaut autant par les corrections et les compléments d'informations qu'il apporte au texte de Nimuendaju (notamment sur les identifications botaniques et zoologiques), que par l'hommage qu'il lui rend en faisant ainsi écho à son goût du détail et du travail méticuleux.

6 Sans doute cet ouvrage n'est-il pas toujours d'une lecture exaltante. On pourrait même en déplorer le caractère souvent austère et parfois désuet. Signalons cependant que ce travers se voit assez régulièrement contrebalancé par les discrètes touches d'humour qui parsèment le texte et à travers lesquelles la personnalité particulièrement attachante de l'auteur transparaît à intervalles réguliers. Les lecteurs moins intéressés par les techniques amérindiennes que par la dimension humaine du travail d'ethnographe retiendront les passages où l'auteur raconte ses déboires avec l'uxorilocalité, la tyrannie des épouses et les caprices des jeunes filles palikur (pp. 106-110), ou encore son effroi 
admiratif devant le spectacle de très jeunes enfants se gorgeant dans l'obscurité la plus profonde de poissons truffés d'arêtes (p. 102). Nimuendaju est certes frustrant quand il se contente de noter sèchement que la flûte turaká « avait été utilisée pendant la guerre contre les Galibi, qui d'ailleurs en étaient les inventeurs » et qu'elle ne servait désormais plus qu'aux jeux de séduction entre jeunes gens (p. 88). On aimerait vraiment en savoir plus sur cet aérophone enchanté, dont «le son sauvagement passionné et plaintif me procurait toujours, particulièrement dans le silence du soir, une émotion profonde " (p.88). Notre auteur se départit également de sa froideur dans ses touchantes descriptions de la noblesse d'âme et de la générosité désintéressée de ses informateurs, pourtant « assez sots pour avoir honte de leur pauvreté » (pp. 107-108). Rien d'étonnant à lire, qu'au moment où il prenait congé, le chef puis le chamane lui aient déclaré n'avoir jamais vu chez eux « un étranger qui les eût si bien traités » (p. 107). Relevons en outre, s'il faut en croire l'auteur (p. 47), qu'à la suite de ses travaux, le gouverneur de l'État de Para a octroyé aux Palikur 25000 ha en propriété commune. Rien que pour cela, ce travail doit être salué comme une réussite.

7 Sur le plan ethnographique, ce livre n'apporte sans doute rien d'absolument exceptionnel. On relèvera cependant des données éparses sur des thèmes rarement abordés, mais qui n'ont pas échappé à l'œil attentif de Nimuendaju: les techniques d'enfumage vespéral (p. 58) ou le recours aux imposantes plaques de granite de la savane pour échapper aux agressions nocturnes des moustiques (p. 60); la composition de la mèche des briquets de quartz et d'acier d'antan (p.59); les lieux d'excrétion et l'utilisation de bâtonnets en guise de torche-culs (p. 59); le badigeonnage des pointes de flèches de bambou pour les protéger contre les insectes xylophages (p.67); les techniques pour mener une pirogue dans la vase en prenant appui sur la perche utilisée comme passerelle (p. 69); le zèle et la ferveur des petites filles à s'adonner à la poterie (p. 75) ; la baignade séparée des hommes et des femmes et l'utilisation de tessons pour brûler les lèvres des jeunes filles, lors du rituel de puberté, afin de les rendre plus discrètes (p.107); l'emploi par le jeune père d'objets miniatures grâce auxquels il emporte l'âme de son enfant avec lui en forêt (y compris à la chasse) (p.112); la récupération de perles dans les urnes anciennes déterrées par hasard (pratique aujourd'hui désuète) (p. 116) ; l'existence ancienne de femmes chamanes (pp. 121-122) ou encore le système de comput permettant de déterminer le jour et l'horaire de début d'une fête au moyen d'une sorte de calendrier de l'avent en bâtonnets à découper (p. 124).

8 En dépit d'un prix qu'on ne saurait qualifier de bon marché, ce livre soigné, magnifiquement mis en page et pourvu d'une iconographie de qualité, est susceptible de toucher un public varié, du touriste à l'universitaire. Les Palikur y trouveront des bribes de leur histoire récente ; les étudiants y découvriront un modèle de rigueur ; historiens, ethnologues et muséologues y dénicheront des pépites. Les linguistes resteront sans doute un peu sur leur faim. Ils trouveront néanmoins de quoi aiguiser leur curiosité, en attendant les prochains ouvrages de cette collection, notamment ceux portant sur les langues. Souhaitons donc bon vent et bonne route à cette Encyclopédie des Guyanes, dont on signalera en conclusion qu'un autre volume vient de paraitre (Grenand 2009). 


\section{BIBLIOGRAPHIE}

\section{GRENAND Françoise}

2009 Encyclopédies palikur, wayana et wayapi. Fascicule 0 : langue, milieu et histoire, Éditions du Comité des travaux historiques et scientifiques/Presses universitaires d'Orléans, Paris/Orléans.

\section{AUTEURS}

\section{PHILIPPE ERIKSON}

Université Paris Ouest Nanterre La Défense 